\title{
Maximal Domains of Preferences Preserving Strategy-Proofness for Generalized Median Voter Schemes*
}

\author{
by \\ Salvador Barberà ${ }^{\dagger}$, Jordi Massó ${ }^{\ddagger}$, and Alejandro Neme ${ }^{\S}$
}

April, 1997

\begin{abstract}
We characterize the maximal sets of preferences under which generalized median voter schemes are strategy-proof. Those domains are defined by a qualified version of single-peakedness, which depends on the distribution of power among agents implied by each generalized median voter scheme.
\end{abstract}

\footnotetext{
*We thank Dolors Berga for helpful comments. Financial support through a grant from the Programa de Cooperación Científica Iberoamericana is acknowledged. The work of S. Barberà and J. Massó is also partially supported by Research Grants PB92-0590 from the Dirección General de Investigación Científica y Técnica, Spanish Ministry of Education, and SGR96-75 from the Comissionat per Universitats i Recerca de la Generalitat de Catalunya.

†Departament d'Economia i d'Història Econòmica and CODE. Universitat Autònoma de Barcelona. 08193, Bellaterra (Barcelona), Spain. E-mail: sbarbera@cc.uab.es

‡Departament d’Economia i d'Història Econòmica and CODE. Universitat Autònoma de Barcelona. 08193, Bellaterra (Barcelona), Spain. E-mail: jmasso@volcano.uab.es

§Instituto de Matemática Aplicada. Universidad Nacional de San Luis and CONICET. Ejército de los Andes 950. 5700, San Luis, Argentina. E-mail: aneme@unsl.edu.ar
} 


\section{Introduction}

This paper investigates the connections between single-peakedness and strategyproofness. Whether or not nontrivial strategy-proof social choice functions exist depends on the environment where we want them to operate. When alternatives can be represented as points in a rectangular grid, and preferences are singlepeaked, generalized median voter schemes are strategy-proof. Outside those situations, nontrivial strategy-proof social choice functions may still exist, but they are harder to find: domain restrictions become less natural.

Single-peakedness of the agent's preferences is often assumed in the existing literature and it is certainly a useful requirement toward the existence of nontrivial strategy-proof social choice functions. In some environments it is sufficient to guarantee it, in others it needs to be combined with additional restrictions. But it is always there. This leads us to investigate, in the present paper, the extent to which some form of single-peakedness might be necessary for strategy-proofness, as well as sufficient.

Our answer is partial, because it only refers to generalized median voter schemes, but it is precise. We start from any such scheme $F$, and we characterize the maximal set of preferences under which $F$ is strategy-proof. It turns out that the condition characterizing this maximal domain is a qualified version of single-peakedness. Previous results in the same vein include Barberà, Sonnenschein and Zhou [4], Serizawa [9], and Barberà, Massó and Serizawa [3]. Our results improve upon these previous results in several directions. We allow for all types of generalized median voter schemes, by not ruling out the existence of vetoers or dummies. We also cover restricted domains under which the range of generalized median voter schemes might not be a cartesian product.

We have chosen to keep this introduction short, leaving further motivational remarks and examples for Section 2, which contains the definitions and a statement of our result. This is proven in Section 3. Section 4 concludes.

\section{Definitions, Notation and the Theorem}

Agents are the elements of a finite set $N=\{1,2, \ldots, n\}$. We assume that $n$ is at least 2 . 
Alternatives are $K$-tuples of integers numbers. For integers $a, b \in Z$, with $a<b$, we will denote the integer interval $[a, b]=\{a, a+1, \ldots, b\}$. A $K$-dimensional box $B$ is a cartesian product of $K$ integer intervals:

$$
B=\prod_{k=1}^{K} B_{k},
$$

where $B_{k}=\left[a_{k}, b_{k}\right]$ and $a_{k}<b_{k}$. A subbox of $B$ is any box $A$ contained in $B$. We endow $B$ with the $L_{1}$-norm. That is, for every $\alpha \in B$,

$$
\|\alpha\|=\sum_{k=1}^{K}\left|\alpha_{k}\right| .
$$

Given $\alpha, \beta \in B$, the minimal box containing $\alpha$ and $\beta$ is defined by

$$
M B(\alpha, \beta)=\{\gamma \in B \mid\|\alpha-\beta\|=\|\alpha-\gamma\|+\|\gamma-\beta\|\} .
$$

Preferences are binary relations on alternatives (or subsets of alternatives). Let $\mathcal{U}$ be the set of complete, transitive and asymmetric preferences on B. Preferences profiles are $n$-tuples of preferences on $B, \mathbf{P} \in \mathcal{U}^{n}$. Preference profiles $\mathbf{P}=\left(P_{1}, \ldots, P_{n}\right)$ are also represented by $\left(P_{i}, P_{-i}\right)$ when we want to stress the role of $i$ 's preference. For $P \in \mathcal{U}$, we denote the alternative most preferred by $P$ as $\tau(P)$, and we call it the top of $P$.

A social choice function on $\widetilde{\mathcal{P}}_{1} \times \ldots \times \widetilde{\mathcal{P}}_{n} \subseteq \mathcal{U}^{n}$ is a function $F: \widetilde{\mathcal{P}}_{1} \times \ldots \times \widetilde{\mathcal{P}}_{n} \rightarrow B$.

The range of a social choice function $F: \widetilde{\mathcal{P}}_{1} \times \ldots \times \widetilde{\mathcal{P}}_{n} \rightarrow B$, is denoted by $R_{F}$. That is,

$$
R_{F}=\left\{\alpha \in B \mid \exists \mathbf{P}=\left(P_{1}, \ldots, P_{n}\right) \in \widetilde{\mathcal{P}}_{1} \times \ldots \times \widetilde{\mathcal{P}}_{n} \text { such that } F(\mathbf{P})=\alpha\right\} .
$$

Social choice functions require each agent to report some preference. A social choice function is strategy-proof if it is always in the best interest of agents to reveal their preferences truthfully. Formally,

Definition 1. A social choice function $F: \widetilde{\mathcal{P}}_{1} \times \ldots \times \widetilde{\mathcal{P}}_{n} \rightarrow B$ is manipulable on $\widetilde{\mathcal{P}}_{1} \times \ldots \times \widetilde{\mathcal{P}}_{n}$ if there exist $\mathbf{P}=\left(P_{1}, \ldots, P_{n}\right) \in \widetilde{\mathcal{P}}_{1} \times \ldots \times \widetilde{\mathcal{P}}_{n}, i \in N$ and $P_{i}^{\prime} \in \widetilde{\mathcal{P}}_{i}$ such that $F\left(P_{i}^{\prime}, P_{-i}\right) P_{i} F(\mathbf{P})$. A social choice function is strategy-proof on $\widetilde{\mathcal{P}}_{1} \times \ldots \times \widetilde{\mathcal{P}}_{n}$ if it is not manipulable on $\widetilde{\mathcal{P}}_{1} \times \ldots \times \widetilde{\mathcal{P}}_{n}$. 
Definition 2. Let $F: \widetilde{\mathcal{P}}_{1} \times \ldots \times \widetilde{\mathcal{P}}_{n} \rightarrow B$ be a social choice function and let $P_{i} \in \widetilde{\mathcal{P}}_{i}$. The set of options left open to the other agents by $i$ declaring $P_{i}$ is defined as follows:

$$
o\left(P_{i}\right)=\left\{\alpha \in R_{F} \mid \text { there exists } P_{-i} \in \widetilde{\mathcal{P}}_{-i} \text { such that } F\left(P_{i}, P_{-i}\right)=\alpha\right\} .
$$

We shall consider different restrictions on preferences, all of them related to single-peakedness. The first one is a natural extension of this classical condition and has been already used in the literature; see for instance Barberà, Gul and Stacchetti [1], Serizawa [9] and Barberà, Massó and Neme [2]. The second one refers to any set $A$ of $K$-dimensional alternatives. The third one involves two sets $A$ and $G(A \subseteq G \subseteq B)$. We present these definitions in sequence for the benefit of the reader, since the first one is very natural while the others are a bit harder to interpret. Formally, though, we need only one of them (Definition 5), which covers the other two as particular cases.

Our first condition is a natural extension of single-peakedness and it coincides with the classical version when $K=1$. It says that whenever alternative $\beta$ is closer than $\gamma$ to the best alternative $\tau(P)$ (lies on the minimal path from $\tau(P)$ to $\gamma$, in the sense of the $L_{1}$-norm) then $\beta P \gamma$.

Definition 3. A preference $P \in \mathcal{U}$ is single-peaked if $\beta P \gamma$ for all $\beta, \gamma \in B(\beta \neq \gamma)$ such that $\beta \in M B(\tau(P), \gamma)$.

Preferences satisfying Definition 3 are characterized by the following two properties. The first one is goodwise single-peakedness: those preferences, restricted to sets of alternatives differing only on one component, are single-peaked. The second is peak-separability: the best alternative for those preferences on such one-dimensional sets are the projection of the global best on the set. ${ }^{1}$

Our next definition involves a subset $A \subseteq B$ and imposes conditions only on elements of this set. It coincides with single-peakedness when $A=B$.

Definition 4. $A$ preference $P \in \mathcal{U}$ is single-peaked on $A \subseteq B$ if $\beta P \gamma$ for all $\beta, \gamma \in A(\beta \neq \gamma)$ such that $\beta \in M B(\tau(P), \gamma)$.

Finally, the third definition refers to two different sets $A$ and $G(A \subseteq G)$. Preferences will be restricted on both sets, but not in the same way. They will

\footnotetext{
${ }^{1}$ Barberà, Gul and Stacchetti [1] introduced first this concept and called it multidimensional single-peaked. Serizawa [9] calls those preferences cross-shaped.
} 
be required to be single-peaked on $A$ and, in addition, to respect some milder restriction on $G \backslash A$. (In applications, $A$ will be a subset of the set of options left open by an agent, under a given social choice function, and $G$ will be a subset of the range of the social choice function).

Definition 5. Consider $A \subseteq G \subseteq B$. A preference $P \in \mathcal{U}$ is single-peaked on $A$ relative to $G$ if for every $\gamma \in G$ and every $\beta \in A \cap M B(\tau(P), \gamma)$ such that $\beta \neq \gamma$ we have that $\beta P \gamma$.

Next, we define generalized median voter schemes. This class of social choice functions are interesting multidimensional extensions of the basic idea of median voting. Additionally, several papers have shown that, in this and similar settings, ${ }^{2}$ they are strategy-proof rules under single-peakedness.

Definition 6. A left (right)-coalition system on $B_{k}=\left[a_{k}, b_{k}\right]$ is a correspondence $\mathcal{W}_{k}$ that assigns to every $\alpha_{k} \in B_{k}$ a collection of nonempty coalitions $\mathcal{W}_{k}\left(\alpha_{k}\right)$ satisfying the following conditions:

(1) If $W \in \mathcal{W}_{k}\left(\alpha_{k}\right)$ and $W \subset W^{\prime}$, then $W^{\prime} \in \mathcal{W}_{k}\left(\alpha_{k}\right)$.

(2) If $\beta_{k}>(<) \alpha_{k}$ and $W \in \mathcal{W}_{k}\left(\alpha_{k}\right)$, then $W \in \mathcal{W}_{k}\left(\beta_{k}\right)$.

(3) $\mathcal{W}_{k}\left(b_{k}\right)=2^{N} \backslash \emptyset\left(\mathcal{W}_{k}\left(a_{k}\right)=2^{N} \backslash \emptyset\right)$.

A family $\mathcal{L}$ of left-coalition systems on $B$ is a collection $\left\{\mathcal{L}_{k}\right\}_{k=1}^{K}$ where each $\mathcal{L}_{k}$ is a left-coalition system on $B_{k}$. Similarly, a family $\mathcal{R}$ of right-coalition systems on $B$ is a collection $\left\{\mathcal{R}_{k}\right\}_{k=1}^{K}$ where each $\mathcal{R}_{k}$ is a right-coalition system on $B_{k}$. Moreover, given a left (right)-coalition system $\mathcal{W}_{k}$ on $B_{k}$ we say that $W \in \mathcal{W}_{k}\left(\alpha_{k}\right)$ is a minimal left (right) coalition if for every $i \in W, W \backslash\{i\} \notin \mathcal{W}_{k}\left(\alpha_{k}\right)$. Given $\mathcal{L}_{k}$ $\left(\mathcal{R}_{k}\right)$ denote by $\mathcal{L}_{k}^{m}\left(\mathcal{R}_{k}^{m}\right)$ the corresponding sets of minimal left (right) coalitions.

For $\mathbf{P}=\left(P_{1}, \ldots, P_{n}\right) \in \mathcal{U}^{n}$ and $\beta_{k} \in B_{k}$, denote by $\tau(\mathbf{P})=\left(\tau\left(P_{1}\right), \ldots, \tau\left(P_{n}\right)\right)$ the vector of tops, and define the coalition to the left (right) of $\beta_{k}$ at $\tau(\mathbf{P})$ by

$$
\begin{aligned}
l\left(\tau(\mathbf{P}), \beta_{k}\right) & =\left\{i \in N \mid \tau_{k}\left(P_{i}\right) \leq \beta_{k}\right\} \\
\left(r\left(\tau(\mathbf{P}), \beta_{k}\right)\right. & \left.=\left\{i \in N \mid \tau_{k}\left(P_{i}\right) \geq \beta_{k}\right\}\right) .
\end{aligned}
$$

Definition 7. Let $\left\{\mathcal{L}_{k}\right\}_{k=1}^{K}\left(\left\{\mathcal{R}_{k}\right\}_{k=1}^{K}\right)$ be a family of left (right)-coalition systems on $B$. The social choice function $F: \widetilde{\mathcal{P}}_{1} \times \ldots \times \widetilde{\mathcal{P}}_{n} \rightarrow B$ is called a Generalized

\footnotetext{
${ }^{2}$ See, for example, Moulin [7], Border and Jordan [6], Barberà, Sonnenschein and Zhou [4], Barberà, Gul and Stacchetti [1], Peters, van der Stel and Storken [8], Barberà, Massó and Neme [2], and Barberà, Massó and Serizawa [3].
} 
Median Voter Scheme $(G M V S)$ defined by $\mathcal{L}(\mathcal{R})$ if it can be defined as follows: for every $\mathbf{P} \in \widetilde{\mathcal{P}}_{1} \times \ldots \times \widetilde{\mathcal{P}}_{n}$ and every $k=1, \ldots, K$

$$
\begin{gathered}
F_{k}(\mathbf{P})=\beta_{k} \Leftrightarrow l\left(\tau(\mathbf{P}), \beta_{k}\right) \in \mathcal{L}_{k}\left(\beta_{k}\right) \text { and } l\left(\tau(\mathbf{P}), \beta_{k}-1\right) \notin \mathcal{L}_{k}\left(\beta_{k}-1\right) \\
\left(F_{k}(\mathbf{P})=\beta_{k} \Leftrightarrow r\left(\tau(\mathbf{P}), \beta_{k}\right) \in \mathcal{R}_{k}\left(\beta_{k}\right) \text { and } r\left(\tau(\mathbf{P}), \beta_{k}+1\right) \notin \mathcal{R}_{k}\left(\beta_{k}+1\right)\right) .
\end{gathered}
$$

Before proceeding, it is useful to understand the relationship between right and left coalition systems, $\mathcal{R}_{k}$ and $\mathcal{L}_{k}$ that produce the same outcome for all $\left(\tau_{k}\left(P_{1}\right), \ldots, \tau_{k}\left(P_{n}\right)\right)$. Given $\mathcal{R}_{k}$, define $\mathcal{L}_{k}^{*}$ as follows: for all $a_{k} \leq \alpha_{k}<b_{k}$,

$$
\begin{aligned}
\mathcal{L}_{k}^{*}\left(\alpha_{k}\right) & =\left\{S \subseteq N \mid S \cap S^{\prime} \neq \emptyset \text { for all } S^{\prime} \in \mathcal{R}_{k}\left(\alpha_{k}+1\right)\right\}, \text { and } \\
\mathcal{L}_{k}^{*}\left(b_{k}\right) & =2^{N} \backslash \emptyset .
\end{aligned}
$$

Remark 1. It is easy to see that $\mathcal{R}_{k}$ and $\mathcal{L}_{k}$ will select the same outcome for all $\left(\tau_{k}\left(P_{1}\right), \ldots, \tau_{k}\left(P_{n}\right)\right)$ if and only if $\mathcal{L}_{k}=\mathcal{L}_{k}^{*}$.

Our definition of generalized median voter schemes induces some distribution of power among agents. Some agents may never be able to influence the outcome at all: they are dummies. Some agents may always dictate the outcome to be in a specific subset: they are decisive. Some agents may avoid some outcomes, if they want: they are vetoers. These possibilities are some times global, but they can also be defined in a local sense: power may depend on the alternative under consideration and also on each of the dimensions defining this alternative. The definition below makes all these notions precise. To do so, let $\mathcal{L}=\left\{\mathcal{L}_{k}\right\}_{k=1}^{K}$ $\left(\mathcal{L}^{m}=\left\{\mathcal{L}_{k}^{m}\right\}_{k=1}^{K}\right)$ be a (minimal) left-coalition system defined on $B$ and let $\mathcal{R}=$ $\left\{\mathcal{R}_{k}\right\}_{k=1}^{K}\left(\mathcal{R}^{m}=\left\{\mathcal{R}_{k}^{m}\right\}_{k=1}^{K}\right)$ and $F: \widetilde{\mathcal{P}}_{1} \times \ldots \times \widetilde{\mathcal{P}}_{n} \rightarrow B$ be its associated (minimal) right-coalition system and generalized median voter scheme, respectively.

Definition 8. We say that agent $i$ is left (right) dummy at $\beta_{k}$ if $i \notin \underset{S \in \mathcal{L}_{k}^{m}\left(\beta_{k}\right)}{\cup} S$ $\left(i \notin \underset{S \in \mathcal{R}_{k}^{m}\left(\beta_{k}\right)}{\cup} S\right)$.

We say that agent $i$ is left (right) vetoer at $\beta_{k}$ if $i \in \underset{S \in \mathcal{L}_{k}\left(\beta_{k}\right)}{\cap} S\left(i \in \underset{S \in \mathcal{R}_{k}\left(\beta_{k}\right)}{\cap} S\right)$. We say that agent $i$ is left (right) decisive at $\beta_{k}$ if $\{i\} \in \mathcal{L}_{k}\left(\beta_{k}\right)\left(\{i\} \in \mathcal{R}_{k}\left(\beta_{k}\right)\right)$. 
Remark 2. The following relationships result from Remark 1.

(1) Assume that $a_{k} \leq \beta_{k}<b_{k}$ : (1.a) if agent $i$ is left dummy at $\beta_{k}$ then agent $i$ is right dummy at $\beta_{k}+1$, and (1.b) if agent $i$ is left vetoer at $\beta_{k}$ then agent $i$ is right decisive at $\beta_{k}+1$.

(2) Assume that $a_{k}<\beta_{k} \leq b_{k}$ : (2.a) if agent $i$ is right dummy at $\beta_{k}$ then agent $i$ is left dummy at $\beta_{k}-1$, and (2.b) if agent $i$ is right vetoer at $\beta_{k}$ then agent $i$ is left decisive at $\beta_{k}-1$.

The definition of a decisive agent follows Serizawa [9]. Notice that its power is weaker than what the name may suggest. If $i$ is left-decisive at $\beta_{k}$, then he can guarantee that the outcome will not be strictly above $\beta_{k}$. In other words, $i$ can veto all values strictly above $\beta_{k}$.

Definition 9. We say that $F$ is a generalized median voter scheme without dummies if for all $k=1, \ldots, K$ the set of left (right) dummies at $\beta_{k}$ is empty for all $\beta_{k} \in\left[a_{k}, b_{k}\right]$.

Our next definition establishes a requirement on the set of feasible preferences.

Definition 10. We say that a domain $\widetilde{\mathcal{P}}_{1} \times \ldots \times \widetilde{\mathcal{P}}_{n}$ is rich on $A \subseteq B$ if for all $i \in N: \alpha \in A \Leftrightarrow \exists P_{i} \in \widetilde{\mathcal{P}}_{i}$ such that $\tau\left(P_{i}\right)=\alpha$.

When $A$ is interpreted as the range of a social choice function this requirement is related with the shape of $A$, in a subtle way, that deserves comment. If $A$ is a cartesian product, then the richness condition simply requires that there should be, for each alternative, at least one admissible preference ranking this alternative as best. This is a standard assumption (see, for instance, Barberà, Sonnenschein, and Zhou [4] and Serizawa [9]). When the set of alternatives is non-cartesian, then the double implication in the definition also implies that only preferences whose top is in the range can be admissible. This is certainly a restriction with bite, which is partly justified in order to avoid circular reasoning when a social choice function is not assumed to be onto. It is not always justifiable though: after all, we are all used to preferring what we can not reach! For a discussion of this point, see Barberà, Massó, and Neme [2]. Yet, since our definition and results apply, in particular, to cartesian ranges, they certainly extend all previous results about maximality. The additional restriction on preferences for the non-cartesian case does not detract from that, and may prove useful in some contexts.

As a starting point, we remind the reader the following result. 
Theorem 1. (Serizawa (1995)) Let $F: \widetilde{\mathcal{P}}_{1} \times \ldots \times \widetilde{\mathcal{P}}_{n} \rightarrow B$ be a strategy-proof Generalized Median Voter Scheme without dummies with rich domain on B. Let $i \in N$. For any $k=1, \ldots, K$, let $d_{k}$ be the maximal level such that $i$ is right decisive in $\mathcal{R}_{k}$, and let $v_{k}$ be the minimal level such that he is a right vetoer in $\mathcal{R}_{k}$. Then any $P_{i} \in \widetilde{\mathcal{P}}_{i}$ is single-peaked on

$$
S\left(P_{i}\right)=\left\{\alpha \in B \mid \forall k=1, \ldots, K, \min \left\{d_{k}, \tau_{k}\left(P_{i}\right)\right\} \leq \alpha_{k} \leq \max \left\{v_{k}, \tau_{k}\left(P_{i}\right)\right\}\right\} .
$$

Our results improve upon this one in several directions. In order to motivate our contributions, let us first rephrase the essential intuition behind Theorem 1 . The set $S\left(P_{i}\right)$ is almost the option set $o\left(P_{i}\right)$, i.e. the set of alternatives that, given that $i$ votes $P_{i}$, may be the final outcome, depending on the votes of others. Precisely, ${ }^{3}$

$$
o\left(P_{i}\right)=\left\{\alpha \in B \mid \forall k=1, \ldots, K, \min \left\{d_{k}, \tau_{k}\left(P_{i}\right)\right\} \leq \alpha_{k} \leq \max \left\{v_{k}-1, \tau_{k}\left(P_{i}\right)\right\}\right\} .
$$

Then, Theorem 1 requires that $i$ 's preferences are single-peaked on $S\left(P_{i}\right)$. This statement is equivalent to requiring that (a) $P_{i}$ is single-peaked on $o\left(P_{i}\right)$, and (b) $v_{k}$ is worse than any point different than $v_{k}$ in $M B\left(\tau_{k}\left(P_{i}\right), v_{k}\right)$ if $v_{k} \neq \tau_{k}\left(P_{i}\right){ }^{4}$ (This rewording may seem artificial, but wait). In fact, single-peakedness on $o\left(P_{i}\right)$ is necessary. But, because agent $i$, by changing his preference from $P_{i}$ to $P_{i}^{\prime}$, can change these options, and shift the outcome, a further requirement is also necessary: other points which might be attained by declaring preferences other than $P_{i}$ must be worse than some points in the option set. Serizawa's condition requires this for the point $v_{k}$ only (and does so implicitly). If we want to get a condition which is not only necessary but also sufficient for strategy-proofness we must require it explicitly and for a (generally) larger set of alternatives.

To be more specific, consider Example 1, which shows that the set of singlepeaked preferences on $S\left(P_{i}\right)$ is still too large in the sense that with those preferences generalized median voter schemes may be manipulable.

Example 1. Consider a one-dimensional problem with four alternatives $\alpha_{1}<$ $\alpha_{2}<\alpha_{3}<\alpha_{4}$, and agents 1 and 2. Define the generalized median voter scheme $F$ as follows: $\mathcal{R}^{m}\left(\alpha_{4}\right)=\{1,2\}$, and $\mathcal{R}^{m}\left(\alpha_{3}\right)=\mathcal{R}^{m}\left(\alpha_{2}\right)=\mathcal{R}^{m}\left(\alpha_{1}\right)=\{\{1\},\{2\}\}$. Notice that $F$ does not have a right-dummy agent and, by Remark 2, it does not have a left-dummy agent. Consider the preference $P_{2}$ of agent 2 such that

\footnotetext{
${ }^{3}$ See Lemma 1 in the Appendix.

${ }^{4}$ This heuristic argument is done assuming implicitly that $K=1$.
} 
$\alpha_{4} P_{2} \alpha_{1} P_{2} \alpha_{2} P_{2} \alpha_{3}$. Since $\alpha_{3}$ is the maximal level such that agent 2 is right decisive in $\mathcal{R}$ and $\alpha_{4}$ is the minimum level such that agent 2 is right vetoer in $\mathcal{R}$, we have that $S\left(P_{2}\right)=\left[\min \left\{\alpha_{3}, \alpha_{4}\right\}, \max \left\{\alpha_{4} \alpha_{4}\right\}\right]=\left[\alpha_{3}, \alpha_{4}\right]$. Since $\alpha_{4} P_{2} \alpha_{3}$ we have that $P_{2}$ is single-peaked on $S\left(P_{2}\right)$. However, to see that agent 2 can manipulate $F$ let $P_{1}$ be any single-peaked preference for agent 1 with the property that $\tau\left(P_{1}\right)=\alpha_{1}$ and $P_{2}^{\prime}=P_{1}$. Then, $F\left(P_{1}, P_{2}^{\prime}\right)=\alpha_{1} P_{2} \alpha_{3}=F\left(P_{1}, P_{2}\right)$.

In view of this, we proceed as follows. We provide necessary and sufficient conditions for strategy-proofness of generalized median voter schemes for the general case where $F$ is not necessarily onto. Before that, in order to allow for better comparison with Serizawa's result and to proceed more smoothly, we state an intermediate result which maintains the non-dummy condition and highlights one of the directions of our extension. Since it will become a Corollary of Theorem 3 (proven in Section 3), we state it without proof.

Theorem 2. Let $F: \widetilde{\mathcal{P}}_{1} \times \ldots \times \widetilde{\mathcal{P}}_{n} \rightarrow B$ be a Generalized Median Voter Scheme with rich domain on $R_{F}$ without dummies. Then, $F$ is strategy-proof on $\widetilde{\mathcal{P}}_{1} \times$ $\ldots \times \widetilde{\mathcal{P}}_{n}$ if and only if for every $i \in N$ and every $P_{i} \in \widetilde{\mathcal{P}}_{i}, P_{i}$ is single-peaked on $o\left(P_{i}\right)$ relative to $R_{F}$.

Example 2 below illustrates that the non-dummy condition in Theorems 1 and 2 is very restrictive because many generalized median voter schemes do not satisfy it. It is obvious that any maximality result should exclude agents which are dummies at all points, but there is a wide gap between the trivial case where an agent is dummy everywhere and those where he might be dummy locally, especially in a multidimensional setting.

Example 2. Consider a one-dimensional problem with ten alternatives $\alpha_{1}<$ $\ldots<\alpha_{10}$, and agents $i$ and $j(i \neq j)$ in a set $N=\{1, \ldots, n\}$ where $n \geq 3$. Define the generalized median voter scheme $F$ as follows: $\mathcal{L}^{m}\left(\alpha_{1}\right)=N \backslash\{i\}, \mathcal{L}^{m}\left(\alpha_{2}\right)=$ $\ldots=\mathcal{L}^{m}\left(\alpha_{9}\right)=\{N \backslash\{i\}, N \backslash\{j\}\}$, and $\mathcal{L}^{m}\left(\alpha_{10}\right)=2^{N} \backslash \emptyset$. Notice that although agent $i$ is only left dummy at $\alpha_{1}, F$ does not satisfy the non-dummy condition, and therefore we can not apply Theorems 1 and 2 .

Consider a generalized median voter scheme $F: \widetilde{\mathcal{P}}_{1} \times \ldots \times \widetilde{\mathcal{P}}_{n} \rightarrow B$ defined by $\mathcal{R}=\left\{\mathcal{R}_{k}\right\}_{k=1}^{K}\left(\right.$ and $\left.\mathcal{L}=\left\{\mathcal{L}_{k}\right\}_{k=1}^{K}\right)$ and let $i \in N$ and $k \in K$ be given. Consider the set of points $\left\{x_{k}^{1}, \ldots, x_{k}^{T}\right\}$ where $a_{k}<x_{k}^{1}<\ldots<x_{k}^{T} \leq b_{k}$ and agent $i$ is right dummy at $x_{k}^{t}$ for all $1 \leq t \leq T$. Denote by $\mathcal{D}_{k}(i)=\left\{\mathcal{D}_{k}^{0}(i), \ldots, \mathcal{D}_{k}^{T}(i)\right\}$ the partition of $\left[a_{k}, b_{k}\right]$ where $\mathcal{D}_{k}^{0}(i)=\left[a_{k}, x_{k}^{1}-1\right], \mathcal{D}_{k}^{t}(i)=\left[x_{k}^{t}, x_{k}^{t+1}-1\right]$ for all $1 \leq t<T$, and $\mathcal{D}_{k}^{T}(i)=\left[x_{k}^{T}, b_{k}\right]$. 
Definition 11. Let $F: \widetilde{\mathcal{P}}_{1} \times \ldots \times \widetilde{\mathcal{P}}_{n} \rightarrow B$ be a generalized median voter scheme defined by $\mathcal{R}=\left\{\mathcal{R}_{k}\right\}_{k=1}^{K}$ (and $\mathcal{L}=\left\{\mathcal{L}_{k}\right\}_{k=1}^{K}$ ) and let $i \in N$. The partition $\mathcal{D}(i)=\prod_{k=1}^{K} \mathcal{D}_{k}(i)$ of $B$ is called the non-dummy partition of $i$.

Theorem 3. Let $F: \widetilde{\mathcal{P}}_{1} \times \ldots \times \widetilde{\mathcal{P}}_{n} \rightarrow B$ be a generalized median voter scheme with rich domain on $R_{F}$. Then, $F$ is strategy -proof on $\widetilde{\mathcal{P}}_{1} \times \ldots \times \widetilde{\mathcal{P}}_{n}$ if and only if for every $i \in N$, every $P_{i} \in \widetilde{\mathcal{P}}_{i}$, and every $D \in \mathcal{D}(i), P_{i}$ is single-peaked on $\left[o\left(P_{i}\right) \cap D\right]$ relative to $\left[R_{F} \cap D\right]$.

Before proving the main result of the paper we illustrate, in Example 3 below, the main concepts used in the definition of single-peaked preferences on $\left[o\left(P_{i}\right) \cap D\right]$ relative to $\left[R_{F} \cap D\right]$.

Example 3. Consider the case with two coordinates where $B=\left\{\alpha_{1}^{1}, \ldots, \alpha_{1}^{7}\right\} \times$ $\left\{\alpha_{2}^{1}, \ldots, \alpha_{2}^{5}\right\}$ and the set of agents is $N=\{1,2,3,4,5\}$. Consider the generalized median voter scheme $F$ is defined by the following family of right-coalition systems $\mathcal{R}=\left\{\mathcal{R}_{1}, \mathcal{R}_{2}\right\}$

$$
\begin{aligned}
& \mathcal{R}_{1}^{m}\left(\alpha_{1}^{7}\right)=\{1,2,3,4,5\}, \\
& \mathcal{R}_{1}^{m}\left(\alpha_{1}^{6}\right)=\{2,3,4,5\} \\
& \mathcal{R}_{1}^{m}\left(\alpha_{1}^{5}\right)=\mathcal{R}_{1}^{m}\left(\alpha_{1}^{4}\right)=\{\{2,3,4,5\},\{1,2,3,4\}\}, \\
& \mathcal{R}_{1}^{m}\left(\alpha_{1}^{3}\right)=\{\{1,2,3\},\{2,3,4\},\{3,4,5\}\}, \\
& \mathcal{R}_{1}^{m}\left(\alpha_{1}^{2}\right)=\{\{2,3\},\{3,4,5\}\}, \\
& \mathcal{R}_{1}^{m}\left(\alpha_{1}^{1}\right)=2^{N} \backslash \emptyset \\
& \mathcal{R}_{2}^{m}\left(\alpha_{2}^{5}\right)=\{1,2,3,4\} \\
& \mathcal{R}_{2}^{m}\left(\alpha_{2}^{4}\right)=\{\{2,3,4\},\{3,4,5\}\}, \\
& \mathcal{R}_{2}^{m}\left(\alpha_{2}^{3}\right)=\mathcal{R}_{2}^{m}\left(\alpha_{2}^{2}\right)=\{\{1,2\},\{3,4\}\}, \text { and } \\
& \mathcal{R}_{2}^{m}\left(\alpha_{2}^{1}\right)=2^{N} \backslash \emptyset .
\end{aligned}
$$

Notice that agent 1 is right dummy at $\alpha_{1}^{2}, \alpha_{1}^{6}$, and $\alpha_{2}^{4}$ but agent 2 is never right dummy. Therefore, the non-dummy partition of agent 1 is $\mathcal{D}(1)=\mathcal{D}_{1}(1) \times \mathcal{D}_{2}(1)$, where $\mathcal{D}_{1}(1)=\left\{\left\{\alpha_{1}^{1}\right\},\left\{\alpha_{1}^{2}, \alpha_{1}^{3}, \alpha_{1}^{4}, \alpha_{1}^{5}\right\},\left\{\alpha_{1}^{6}, \alpha_{1}^{7}\right\}\right\}$ and $\mathcal{D}_{2}(1)=\left\{\left\{\alpha_{2}^{1}, \alpha_{2}^{2}, \alpha_{2}^{3}\right\},\left\{\alpha_{2}^{4}, \alpha_{2}^{5}\right\}\right\}$, while the non-dummy partition of agent 2 is the box $B$ itself since $\mathcal{D}_{1}(2)=$ $\left\{\left\{\alpha_{1}^{1}, \ldots, \alpha_{1}^{7}\right\}\right\}$ and $\mathcal{D}_{2}(2)=\left\{\left\{\alpha_{2}^{1}, \ldots, \alpha_{2}^{5}\right\}\right\}$. Consider any set of preferences $\widetilde{\mathcal{P}}_{1} \times$ $\ldots \times \widetilde{\mathcal{P}}_{5}$ for which $F$ has rich domain on $B\left(=R_{F}\right)$. Notice that since $\mathcal{D}(2)=\{B\}$, 
any single-peaked preference $P_{2}$ on $o\left(P_{2}\right)$ is indeed single-peaked on $\left[o\left(P_{2}\right) \cap D\right]$ relative to $\left[R_{F} \cap D\right]$ because $\left[o\left(P_{2}\right) \cap D\right]=o\left(P_{2}\right)$ and $\left[R_{F} \cap D\right]=B$. Consider any preference $P_{1} \in \widetilde{\mathcal{P}}_{1}$ such that $\tau\left(P_{1}\right)=\left(\alpha_{1}^{3}, \alpha_{2}^{2}\right)$. Notice that $o\left(P_{1}\right)=$ $\left\{\alpha_{1}^{1}, \alpha_{1}^{2}, \alpha_{1}^{3}, \alpha_{1}^{4}, \alpha_{1}^{5}, \alpha_{1}^{6}\right\} \times\left\{\alpha_{2}^{1}, \alpha_{2}^{2}, \alpha_{2}^{3}, \alpha_{2}^{4}\right\}$. If $F$ is strategy-proof we must have, for instance that $\left(\alpha_{1}^{6}, \alpha_{2}^{2}\right) P_{1}\left(\alpha_{1}^{7}, \alpha_{2}^{1}\right)$ and $\left(\alpha_{1}^{4}, \alpha_{2}^{3}\right) P_{1}\left(\alpha_{1}^{5}, \alpha_{2}^{3}\right)$ but we could have either $\left(\alpha_{1}^{5}, \alpha_{2}^{5}\right) P_{1}\left(\alpha_{1}^{4}, \alpha_{2}^{3}\right)$ or $\left(\alpha_{1}^{4}, \alpha_{2}^{3}\right) P_{1}\left(\alpha_{1}^{5}, \alpha_{2}^{5}\right)$ since $\left(\alpha_{1}^{5}, \alpha_{2}^{5}\right)$ and $\left(\alpha_{1}^{4}, \alpha_{2}^{3}\right)$ belong to different elements of the non-dummy partition of agent 1 .

\section{Proof of Theorem 3}

Let $F: \widetilde{\mathcal{P}}_{1} \times \ldots \times \widetilde{\mathcal{P}}_{n} \rightarrow B$ be a Generalized Median Voter Scheme defined by $\mathcal{R}=\left\{\mathcal{R}_{k}\right\}_{k=1}^{K}\left(\right.$ and $\mathcal{L}=\left\{\mathcal{L}_{k}\right\}_{k=1}^{K}$ ) with rich domain on $R_{F}$. Let $i \in N, k=1, \ldots, K$ and $P_{i}$ be a preference ordering in $\widetilde{\mathcal{P}}_{i}$. Define:

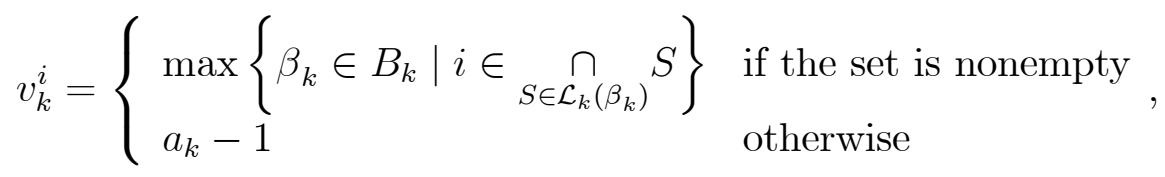

$$
\begin{aligned}
& d_{k}^{i}=\min \left\{\beta_{k} \in B_{k} \mid\{i\} \in \mathcal{L}_{k}\left(\beta_{k}\right)\right\}, \\
& a_{k}\left(P_{i}\right)=\min \left\{v_{k}^{i}+1, \tau_{k}\left(P_{i}\right)\right\}, \\
& b_{k}\left(P_{i}\right)=\max \left\{d_{k}^{i}, \tau_{k}\left(P_{i}\right)\right\} \text {, and } \\
& A\left(P_{i}\right)=R_{F} \cap\left[\prod_{k=1}^{K}\left[a_{k}\left(P_{i}\right), b_{k}\left(P_{i}\right)\right]\right] .^{5}
\end{aligned}
$$

Lemma 1 below describes, for any given generalized median voter scheme, the exact shape of the set of options left open by an agent $i$ to the other agents.

Lemma 1. $A\left(P_{i}\right)=o\left(P_{i}\right)$.

\footnotetext{
${ }^{5}$ Notice that $a_{k}$ and $b_{k}$ were already defined as the extreme values in the range of the function. The values $a_{k}\left(P_{i}\right)$ and $b_{k}\left(P_{i}\right)$ are defined here. We keep a parallel notation, since $\left[a_{k}\left(P_{i}\right), b_{k}\left(P_{i}\right)\right]$ will again stand for intervals defined by their extrems.
} 
Proof. Let $\beta \in A\left(P_{i}\right)$. For every $j \neq i$, consider any $P_{j} \in \widetilde{\mathcal{P}}_{j}$ with the property that $\tau\left(P_{j}\right)=\beta$. We will show that $F\left(P_{i}, P_{-i}\right)=\beta$. Let $k=1, \ldots, K$ be arbitrary and define the set $S=\left\{j \in N \mid \tau_{k}\left(P_{j}\right) \leq \beta_{k}\right\}$; by construction, $N \backslash\{i\} \subseteq S$. First, suppose that $i \notin S$. Then, $v_{k}^{i}+1 \leq \beta_{k}$, since $a_{k}\left(P_{i}\right) \leq$ $\beta_{k}<\tau_{k}\left(P_{i}\right)$ implies that $a_{k}\left(P_{i}\right)=v_{k}^{i}+1$. Therefore, $S \in \mathcal{L}_{k}\left(\beta_{k}\right)$. Moreover, $\left\{j \in N \mid \tau_{k}\left(P_{j}\right) \leq \beta_{k}-1\right\}=\emptyset$, which implies that $F_{k}\left(P_{i}, P_{-i}\right)=\beta_{k}$. Second, assume that $i \in S$; that is $S=N$, which implies that $S \in \mathcal{L}_{k}\left(\beta_{k}\right)$. By construction, the set $\bar{S}=\left\{j \in N \mid \tau_{k}\left(P_{j}\right) \leq \beta_{k}-1\right\}$ is either empty or is equal to the set $\{i\}$. Suppose $\bar{S}=\{i\}$, then $\beta_{k} \leq d_{k}^{i}$ since $\tau_{k}\left(P_{i}\right)<\beta_{k}$ and $b_{k}\left(P_{i}\right)=\max \left\{d_{k}^{i}, \tau_{k}\left(P_{i}\right)\right\}$ imply that $b_{k}\left(P_{i}\right)=d_{k}^{i}$. From the hypothesis that $\beta_{k} \leq b_{k}\left(P_{i}\right)$ it follows that $\beta_{k}-1<d_{k}^{i}$. Therefore $\{i\} \notin \mathcal{L}_{k}\left(\beta_{k}-1\right)$ which implies that $F_{k}\left(P_{i}, P_{-i}\right)=\beta_{k}$. Since $k \in K$ was arbitrary, we have that $\beta \in o\left(P_{i}\right)$.

Let $\alpha \in o\left(P_{i}\right)$. That is, there exists $P_{-i} \in \widetilde{\mathcal{P}}_{-i}$ such that $F\left(P_{i}, P_{-i}\right)=\alpha$. Define $\mathbf{P}=\left(P_{i}, P_{-i}\right)$. Let $k=1, \ldots, K$ be arbitrary. Notice that if $\tau_{k}\left(P_{i}\right)=\alpha_{k}$ the result follows immediately by (3.3) and (3.4). Assume first that $\tau_{k}\left(P_{i}\right)<\alpha_{k}$. It implies that $a_{k}\left(P_{i}\right)<\alpha_{k}$. Define the set $S=\left\{j \in N \mid \tau_{k}\left(P_{j}\right) \leq \alpha_{k}-1\right\}$. Since $F(\mathbf{P})=\alpha$ we know that $S \notin \mathcal{L}_{k}\left(\alpha_{k}-1\right)$. However, since $i \in S$ we have that $\alpha_{k}-1<d_{k}^{i}$ implying that $\alpha_{k} \leq b_{k}\left(P_{i}\right)$ since $\tau_{k}\left(P_{i}\right)<\alpha_{k} \leq d_{k}^{i}$ and (3.4) hold. Assume now that $\tau_{k}\left(P_{i}\right)>\alpha_{k}$. It implies that $\alpha_{k}<b_{k}\left(P_{i}\right)$. Define the set $S=\left\{j \in N \mid \tau_{k}\left(P_{j}\right) \leq \alpha_{k}\right\}$ which belongs to $\mathcal{L}_{k}\left(\alpha_{k}\right)$ since $F_{k}(\mathbf{P})=\alpha_{k}$. Notice that $i \notin S$ which means that $v_{k}^{i}+1 \leq \alpha_{k}$. Therefore, (3.3) and $v_{k}^{i}+1 \leq \alpha_{k}<\tau_{k}\left(P_{i}\right)$ imply that $a_{k}\left(P_{i}\right)=v_{k}^{i}+1$. Hence, $a_{k}\left(P_{i}\right) \leq \alpha_{k}$. Since $k \in K$ was arbitrary, we have that $\alpha \in A\left(P_{i}\right)$.

\subsection{Necessity}

Let $F$ be strategy-proof on $\widetilde{\mathcal{P}}_{1} \times \ldots \times \widetilde{\mathcal{P}}_{n}$. Consider $i \in N, P_{i} \in \widetilde{\mathcal{P}}_{i}$, and $D \in \mathcal{D}(i)$. Let $\gamma \in R_{F} \cap D$ and $\beta \in A\left(P_{i}\right) \cap D \cap M B\left(\gamma, \tau\left(P_{i}\right)\right)$ be such that $\beta \neq \gamma$.

Let $K_{1}=\left\{k \in K \mid \gamma_{k}<\beta_{k} \leq \tau_{k}\left(P_{i}\right)\right\}$ and $K_{2}=\left\{k \in K \mid \tau_{k}\left(P_{i}\right) \leq \beta_{k}<\gamma_{k}\right\}$. Notice that $K_{1} \cup K_{2} \neq \emptyset$ since $\beta \neq \gamma$ and $\beta \in M B\left(\gamma, \tau\left(P_{i}\right)\right)$.

Lemma 2. If $A\left(P_{i}\right) \cap D \cap M B(\gamma, \beta)=\{\gamma, \beta\}$ then $\beta P_{i} \gamma$.

Proof. The proof is based on the choice of a profile such that, when $i$ declares his top $\tau\left(P_{i}\right)$, then $\beta$ obtains, but $i$ could change the outcome to $\gamma$ by voting for $\gamma$. To find such profile, we will divide the proof into two different cases.

Case 1: Assume that $K_{1} \neq \emptyset$. That is, there exists $\tilde{k}$ such that $\gamma_{\tilde{k}}<\beta_{\tilde{k}} \leq$ $\tau_{\tilde{k}}\left(P_{i}\right)$ and $i$ is not a left dummy at $\gamma_{\tilde{k}}$ because $\gamma_{\tilde{k}}, \beta_{\tilde{k}} \in\left[x_{\tilde{k}}^{t}, x_{\tilde{k}}^{t+1}-1\right]$ for some 
$0 \leq t \leq T_{\tilde{k}}$ and $\gamma_{\tilde{k}}<\beta_{\tilde{k}}$. Let $S \subseteq N$ be such that $i \in S \in \mathcal{L}_{\tilde{k}}^{m}\left(\gamma_{\tilde{k}}\right)$, and consider $P_{-i}$ where for every $j \in N \backslash\{i\}, P_{j} \in \widetilde{\mathcal{P}}_{j}$ is such that

$$
\tau\left(P_{j}\right)=\left\{\begin{array}{ll}
\gamma & \text { if } j \in S \backslash\{i\} \\
\beta & \text { if } j \in N \backslash S
\end{array},\right.
$$

which exist since $\beta, \gamma \in R_{F}$ and $F$ has rich domain on $R_{F}$.

First, for every $k \notin K_{1} \cup K_{2}$ we have that $N \backslash\{i\} \subset\left\{j \in N \mid \tau_{k}\left(P_{j}\right) \leq \beta_{k}=\gamma_{k}\right\}$. Using the fact that $\beta, \gamma \in A\left(P_{i}\right)$ we will show that $F_{k}\left(P_{i}, P_{-i}\right)=\gamma_{k}=\beta_{k}$. To see it, first assume that $F_{k}\left(P_{i}, P_{-i}\right)<\gamma_{k}=\beta_{k}$, which would imply that $\{i\} \in \mathcal{L}_{k}\left(F_{k}\left(P_{i}, P_{-i}\right)\right)$ and $\tau_{k}\left(P_{i}\right) \leq \gamma_{k}=\beta_{k}$; but from these two conditions we could conclude that $b_{k}\left(P_{i}\right)=\max \left\{d_{k}^{i}, \tau_{k}\left(P_{i}\right)\right\}<\gamma_{k}=\beta_{k}$ contradicting the hypothesis that $\beta, \gamma \in A\left(P_{i}\right)$. Assume now that $F_{k}\left(P_{i}, P_{-i}\right)>\gamma_{k}=\beta_{k}$, which would imply that $\tau_{k}\left(P_{i}\right)>\gamma_{k}=\beta_{k}$. Therefore, $N \backslash\{i\} \notin \mathcal{L}_{k}\left(\gamma_{k}\right)$ implying that $i \in \bigcap_{S \in \mathcal{L}_{k}\left(\gamma_{k}\right)} S$, which would mean that $v_{k}^{i}+1>\gamma_{k}=\beta_{k}$. Therefore, we would have that $\gamma_{k}=\beta_{k}<\min \left\{v_{k}^{i}+1, \tau_{k}\left(P_{i}\right)\right\}=a_{k}\left(P_{i}\right)$, contradicting the hypothesis that $\beta, \gamma \in A\left(P_{i}\right)$. Hence,

$$
F_{k}\left(P_{i}, P_{-i}\right)=\beta_{k} \text { for all } k \notin K_{1} \cup K_{2} \text {. }
$$

Second, for every $k \in K_{1}$ the set $\left\{j \in N \mid \tau_{k}\left(P_{j}\right) \leq \beta_{k}\right\}$ contains the set $N \backslash$ $\{i\}$. Since $\beta \in A\left(P_{i}\right)$ by hypothesis, $N \backslash\{i\} \in \mathcal{L}_{k}\left(\beta_{k}\right)$ and therefore $F_{k}\left(P_{i}, P_{-i}\right) \leq$ $\beta_{k}$. Moreover, $\gamma_{k} \leq F_{k}\left(P_{i}, P_{-i}\right)$ because $\tau_{k}\left(P_{j}\right) \geq \gamma_{k}$ for every $j \in N$. Hence,

$$
\gamma_{k} \leq F_{k}\left(P_{i}, P_{-i}\right) \leq \beta_{k} \text { for all } k \in K_{1} .
$$

The set $\left\{j \in N \mid \tau_{\tilde{k}}\left(P_{j}\right) \leq \gamma_{\tilde{k}}\right\}$ is equal to $S \backslash\{i\}$. Since $S \backslash\{i\} \notin \mathcal{L}_{\tilde{k}}\left(\gamma_{\tilde{k}}\right)$ we must have that

$$
\gamma_{\tilde{k}}<F_{\tilde{k}}\left(P_{i}, P_{-i}\right) .
$$

Third, for every $k \in K_{2}$ the set $\left\{j \in N \mid \tau_{k}\left(P_{j}\right)<\beta_{k}\right\}$ is either empty, in which case $F_{k}\left(P_{i}, P_{-i}\right) \geq \beta_{k}$, or else it is equal to the set $\{i\}$. But since $\beta \in A\left(P_{i}\right)$ implies that $\{i\} \notin \mathcal{L}_{k}\left(\beta_{k}-1\right)$ we must have that $F_{k}\left(P_{i}, P_{-i}\right) \geq \beta_{k}$. Hence,

$$
F_{k}\left(P_{i}, P_{-i}\right) \geq \beta_{k} \text { for all } k \in K_{2} .
$$

It is straightforward to see that from (3.6), (3.7), (3.8), (3.9), and the hypothesis of Lemma 2 it follows that $F\left(P_{i}, P_{-i}\right)=\beta$. 
Consider any $\bar{P}_{i} \in \widetilde{\mathcal{P}}_{i}$ with the property that $\tau\left(\bar{P}_{i}\right)=\gamma$, which exists since $\gamma \in R_{F}$ and $F$ has rich domain on $R_{F}$. Now, $F\left(\bar{P}_{i}, P_{-i}\right) \in M B(\beta, \gamma)$ because for every $j \in N$ we have that $\tau\left(P_{j}\right) \in\{\beta, \gamma\}$. Consider again the coordinate $\tilde{k} \in K_{1}$ and the set $S=\left\{j \in N \mid \tau_{\tilde{k}}\left(P_{j}\right) \leq \gamma_{\tilde{k}}\right\}$, which belongs to $\mathcal{L}_{\tilde{k}}^{m}\left(\gamma_{\tilde{k}}\right)$. Therefore, $F_{\tilde{k}}\left(\bar{P}_{i}, P_{-i}\right)=\gamma_{\tilde{k}}$, which implies, by the hypothesis of Lemma 2 , that $F\left(\bar{P}_{i}, P_{-i}\right)=\gamma$. Since $F$ is strategy-proof on $\widetilde{\mathcal{P}}_{1} \times \ldots \times \widetilde{\mathcal{P}}_{n}$ we must have that $\beta P_{i} \gamma$.

Case 2: Assume that $K_{1}=\emptyset$ and $K_{2} \neq \emptyset$. That is, there exists $\tilde{k}$ such that $\gamma_{\tilde{k}}>\beta_{\tilde{k}} \geq \tau_{\tilde{k}}\left(P_{i}\right)$. Notice that $i$ is not a right dummy at $\gamma_{\tilde{k}}$ because $\gamma_{\tilde{k}}, \beta_{\tilde{k}} \in\left[x_{\tilde{k}}^{t}, x_{\tilde{k}}^{t+1}-1\right]$ for some $0 \leq t \leq T_{\tilde{k}}$ and $\gamma_{\tilde{k}}>\beta_{\tilde{k}}$. Let $S \subseteq N$ be such that $i \in S \in \mathcal{R}_{\tilde{k}}^{m}\left(\gamma_{\tilde{k}}\right)$, and consider $P_{-i}$ where for every $j \in N \backslash\{i\}, P_{j} \in \widetilde{\mathcal{P}}_{j}$ is such that

$$
\tau\left(P_{j}\right)=\left\{\begin{array}{ll}
\gamma & \text { if } j \in S \backslash\{i\} \\
\beta & \text { if } j \in N \backslash S
\end{array},\right.
$$

which exist since $\beta, \gamma \in R_{F}$ and $F$ has rich domain on $R_{F}$.

First, for every $k \notin K_{2}$ we have that $F_{k}\left(P_{i}, P_{-i}\right)=\gamma_{k}=\beta_{k}$ since $N \backslash\{i\} \subset$ $\left\{j \in N \mid \tau_{k}\left(P_{i}\right) \geq \beta_{k}=\gamma_{k}\right\}$ and $i$ is neither a right-decisive nor a right-vetoer agent at $\beta_{k}=\gamma_{k}$. Therefore,

$$
F_{k}\left(P_{i}, P_{-i}\right)=\beta_{k} \text { for all } k \notin K_{2} .
$$

Second, for every $k \in K_{2}$ the set $\left\{j \in N \mid \tau_{k}\left(P_{j}\right) \geq \beta_{k}\right\}$ contains the set $N \backslash\{i\}$. Since $i$ is not a right-vetoer agent at $\beta_{k}$ (remember that $\beta \in A\left(P_{i}\right)$ ), we have that $N \backslash\{i\} \in \mathcal{R}_{k}\left(\beta_{k}\right)$. Therefore,

$$
F_{k}\left(P_{i}, P_{-i}\right) \geq \beta_{k} \text { for all } k \in K_{2}
$$

Moreover, the set $\left\{j \in N \mid \tau_{\tilde{k}}\left(P_{j}\right) \geq \gamma_{\tilde{k}}\right\}$ is equal to $S \backslash\{i\}$. Since $S \backslash\{i\} \notin \mathcal{R}_{\tilde{k}}\left(\gamma_{\tilde{k}}\right)$ we must have that

$$
\gamma_{\tilde{k}}>F_{\tilde{k}}\left(P_{i}, P_{-i}\right)
$$

It is straightforward to see that from (3.10), (3.11), (3.12) and the hypothesis of Lemma 2 it follows that $F\left(P_{i}, P_{-i}\right)=\beta$.

Consider any $\bar{P}_{i} \in \widetilde{\mathcal{P}}_{i}$ with the property that $\tau\left(\bar{P}_{i}\right)=\gamma$, which exists since $\gamma \in R_{F}$ and $F$ has rich domain on $R_{F}$. Now, $F\left(P_{i}, P_{-i}\right) \in M B(\beta, \gamma)$ because for every $j \in N$ we have that $\tau\left(P_{j}\right) \in\{\beta, \gamma\}$. Consider again the coordinate $\tilde{k} \in K_{2}$ and the set $S=\left\{j \in N \mid \tau_{\tilde{k}}\left(P_{j}\right) \geq \gamma_{\tilde{k}}\right\}$, which belongs to $\mathcal{R}_{\tilde{k}}^{m}\left(\gamma_{\tilde{k}}\right)$. 
Therefore, $F_{\tilde{k}}\left(\bar{P}_{i}, P_{-i}\right)=\gamma_{\tilde{k}}$, which implies, by the hypothesis of Lemma 2 , that $F\left(\bar{P}_{i}, P_{-i}\right)=\gamma$. Since $F$ is strategy-proof on $\widetilde{\mathcal{P}}_{1} \times \ldots \times \widetilde{\mathcal{P}}_{n}$ we must have that $\beta P_{i} \gamma$.

Lemma 3. If $A\left(P_{i}\right) \cap D \cap M B(\gamma, \beta) \supsetneq\{\gamma, \beta\}$ then $\beta P_{i} \gamma$.

Proof. Given $\gamma$ and $\beta$, there will exist $\alpha_{1}=\beta, \alpha_{2}, \ldots, \alpha_{h-1}, \alpha_{h}=\gamma$ such that, for each $j, A\left(P_{i}\right) \cap D \cap M B\left(\alpha_{j}, \alpha_{j+1}\right)=\left\{\alpha_{j}, \alpha_{j+1}\right\}$. Specifically, we can choose such $\alpha_{j}$ 's by letting $\alpha_{j+1}$ be one of the closest elements (in the $L_{1}$-norm) to $\alpha_{j}$ in $A\left(P_{i}\right) \cap D \cap M B\left(\alpha_{j}, \gamma\right)$. Now, to prove Lemma 3, apply successively Lemma 2 and the transitivity of the preference ordering $P_{i}$.

Lemma 4. If $\gamma \notin A\left(P_{i}\right)$ then $\beta P_{i} \gamma$.

Proof. For each $j \in N$ consider any $\bar{P}_{j} \in \widetilde{\mathcal{P}}_{j}$ such that $\tau\left(\bar{P}_{j}\right)=\gamma$, which exists since $\gamma \in R_{F}$ and $F$ has rich domain on $R_{F}$. Obviously, $F\left(\bar{P}_{1}, \ldots, \bar{P}_{n}\right)=\gamma$. The proof will consists of two steps.

Step 1: We want to show that $a_{k}\left(P_{i}\right) \leq F_{k}\left(P_{i}, \bar{P}_{-i}\right) \leq b_{k}\left(P_{i}\right)$ for all $k=$ $1, \ldots, K$. But this is immediate, because by definition of option set $F\left(P_{i}, \bar{P}_{-i}\right) \in$ $o\left(P_{i}\right)$, and by Lemma 1 , we have that $a_{k}\left(P_{i}\right) \leq F_{k}\left(P_{i}, \bar{P}_{-i}\right) \leq b_{k}\left(P_{i}\right)$ for all $k=1, \ldots, K$.

Step 2: We want to show that for all $k=1, \ldots, K$ :

(1) if $\left(\tau_{k}\left(P_{i}\right) \leq\right) \beta_{k} \leq \gamma_{k}$ then $\beta_{k} \leq F_{k}\left(P_{i}, \bar{P}_{-i}\right) \leq \gamma_{k}$,

(2) if $\gamma_{k}<\beta_{k}\left(\leq \tau_{k}\left(P_{i}\right)\right)$ then $\gamma_{k} \leq F_{k}\left(P_{i}, \bar{P}_{-i}\right) \leq \beta_{k}$.

Define $\hat{\mathbf{P}}=\left(P_{i}, \bar{P}_{-i}\right)$. To show (1) assume that $\beta_{k} \leq \gamma_{k}$ and notice that the set $\left\{j \in N \mid \tau_{k}(\hat{P} j) \leq \gamma_{k}\right\}$ is equal to $N$. Therefore, $F_{k}(\hat{\mathbf{P}}) \leq \gamma_{k}$. If $\beta_{k}=a_{k}$ then $\beta_{k} \leq F_{k}(\hat{\mathbf{P}})$. Assume that $a_{k}<b_{k}$. Since $\beta \in A\left(P_{i}\right)$ we know that $\beta_{k} \in\left[a_{k}\left(P_{i}\right), b_{k}\left(P_{i}\right)\right]$, which implies that $\beta_{k} \leq d_{k}^{i}$ because $\tau_{k}\left(P_{i}\right) \leq \beta_{k}$. Therefore, $\{i\} \notin \mathcal{L}_{k}\left(\beta_{k}-1\right)$. Moreover, since $\left\{j \in N \mid \beta_{k}>\tau_{k}\left(\hat{P}_{j}\right)\right\} \subseteq\{i\}$ we must have that $\beta_{k} \leq F_{k}(\hat{\mathbf{P}})$. To show (2), assume that $\gamma_{k}<\beta_{k}$ and notice that the set $\left\{j \in N \mid \gamma_{k} \leq \tau_{k}\left(\hat{P}_{j}\right)\right\}$ is equal to $N$. This implies that $\gamma_{k} \leq F_{k}(\hat{\mathbf{P}})$. Since $\beta \in A\left(P_{i}\right)$ we know that $a_{k}\left(P_{i}\right) \leq \beta_{k}$, but because $\tau_{k}\left(P_{i}\right) \geq \beta_{k}$, we must have 
that $\beta_{k}>v_{k}^{i}$, implying that there exists $S \in \mathcal{L}_{k}^{m}\left(\beta_{k}\right)$ such that $i \notin S$. Since $\left\{j \in N \mid \beta_{k} \geq \tau_{k}\left(\hat{P}_{j}\right)\right\} \supseteq N \backslash\{i\} \supseteq S$ it follows that $F_{k}(\hat{\mathbf{P}}) \leq \beta_{k}$.

From (1) and (2) we have established that $\gamma \neq F\left(P_{i}, \bar{P}_{-i}\right) \in A\left(P_{i}\right) \cap M B(\beta, \gamma)$. Since $\{\beta, \gamma\} \subset D$, from (1) and (2) we have that $F\left(P_{i}, \bar{P}_{-i}\right) \in D$. Moreover, since $F$ is strategy-proof and $F(\overline{\mathbf{P}})=\gamma$ we must have $F\left(P_{i}, \bar{P}_{-i}\right) P_{i} \gamma$. Define $F\left(P_{i}, \bar{P}_{-i}\right)=\gamma^{\prime}$. Notice that $\beta \in M B\left(\gamma^{\prime}, \tau\left(P_{i}\right)\right)$ and $\gamma^{\prime} \in A\left(P_{i}\right) \cap D$, implying that the hypothesis of either Lemma 2 or Lemma 3 is satisfied. Therefore, we can deduce that $\beta P_{i} \gamma^{\prime}$ and by transitivity of $P_{i}$ we can conclude that $\beta P_{i} \gamma$.

\subsection{Sufficiency}

Assume that $F$ is not strategy-proof. Then, there exist $i \in N, \mathbf{P}=\left(P_{1}, \ldots, P_{n}\right) \in$ $\widetilde{\mathcal{P}}_{1} \times \ldots \times \widetilde{\mathcal{P}}_{n}$ and $P_{i}^{\prime} \in \widetilde{\mathcal{P}}_{i}$ such that

$$
F\left(P_{i}^{\prime}, P_{-i}\right) P_{i} F(\mathbf{P}) .
$$

Denote by $\overline{\mathbf{P}}$ the profile $\left(P_{i}^{\prime}, P_{-i}\right)$ and let $\gamma=F(\overline{\mathbf{P}})$ and $\beta=F(\mathbf{P})$. We want to show that there exists $D \in \mathcal{D}(i)$ such that $\beta \in A\left(P_{i}\right) \cap D \cap M B\left(\gamma, \tau\left(P_{i}\right)\right)$ and $\gamma \in R_{F} \cap D$. First, notice that $\beta=F\left(P_{i}, P_{-i}\right)$ implies that $\beta \in o\left(P_{i}\right)$, and therefore, by Lemma 1 , we have that $\beta \in A\left(P_{i}\right)$.

Lemma 5. $\beta \in M B\left(\gamma, \tau\left(P_{i}\right)\right)$

Proof. To show it, assume first that $\beta_{k}<\tau_{k}\left(P_{i}\right)$. We will show that $\gamma_{k} \leq \beta_{k}$. Since $F_{k}(\mathbf{P})=\beta_{k}$ we have that $S=\left\{j \in N \mid \tau_{k}\left(P_{j}\right) \leq \beta_{k}\right\} \in \mathcal{L}_{k}\left(\beta_{k}\right)$ and because $i \notin S$ we have that $S \subseteq\left\{j \in N \mid \tau_{k}\left(\bar{P}_{j}\right) \leq \beta_{k}\right\} \in \mathcal{L}_{k}\left(\beta_{k}\right)$ by condition (1) in the definition of a left-coalition system. Then, clearly $F_{k}(\overline{\mathbf{P}}) \leq \beta_{k}$ which is the desired result because $\gamma_{k}=F_{k}(\overline{\mathbf{P}})$. Assume that $\beta_{k}>\tau_{k}\left(P_{i}\right)$. We will show that $\beta_{k} \leq \gamma_{k}$. Since $F_{k}(\mathbf{P})=\beta_{k}$, the set $S=\left\{j \in N \mid \tau_{k}\left(P_{j}\right) \leq \beta_{k}-1\right\} \notin \mathcal{L}_{k}\left(\beta_{k}-1\right)$ and because $i \in S$ we have that $\left\{j \in N \mid \tau_{k}\left(\bar{P}_{j}\right) \leq \beta_{k}-1\right\} \subseteq S \notin \mathcal{L}_{k}\left(\beta_{k}-1\right)$ implying that $\gamma_{k}=F_{k}(\overline{\mathbf{P}}) \geq \beta_{k}$. Finally, if $\beta_{k}=\tau_{k}\left(P_{i}\right)$ we do not have to prove anything since the minimal box condition for dimension $k$ is irrelevant; that is, $\gamma_{k}$ could be both higher or smaller than $\beta_{k}=\tau_{k}\left(P_{i}\right)$.

Lemma 6. There exists $D \in \mathcal{D}(i)$ such that $\{\gamma, \beta\} \subset D$.

Proof. We have to show that:

(1) If $\gamma_{k}<\beta_{k}$ then $i$ is not left dummy at $\xi$ for every $\gamma_{k} \leq \xi<\beta_{k}$, and

(2) If $\beta_{k}<\gamma_{k}$ then $i$ is not right dummy at $\xi$ for every $\beta_{k}<\xi \leq \gamma_{k}$. 
We will show only (1), since the argument to show (2) is the symmetric one using right instead of left coalitions. Assume $\gamma_{k}<\beta_{k}\left(\leq \tau_{k}\left(P_{i}\right)\right)$. The inequality $\beta_{k} \leq \tau_{k}\left(P_{i}\right)$ follows from Lemma 5. By condition (3.13) the coalition $S=$ $\left\{j \in N \mid \tau_{k}\left(P_{j}\right) \leq \gamma_{k}\right\}=\left\{j \in N \backslash\{i\} \mid \tau_{k}\left(\bar{P}_{j}\right) \leq \gamma_{k}\right\}$ is not a member of $\mathcal{L}_{k}\left(\gamma_{k}\right)$ since $\gamma_{k}<\beta_{k}=F_{k}(\mathbf{P})$. However, $\bar{S}=\left\{j \in N \mid \tau_{k}\left(\bar{P}_{j}\right) \leq \gamma_{k}\right\} \in \mathcal{L}_{k}\left(\gamma_{k}\right)$ since $F_{k}(\overline{\mathbf{P}})=\gamma_{k}$ implying that $i \in \bar{S}$ and $\bar{S} \backslash\{i\}=S \notin \mathcal{L}_{k}\left(\gamma_{k}\right)$ which in turn implies that there exists $T \subseteq \bar{S}$ such that $i \in T$ and $T \in \mathcal{L}_{k}^{m}\left(\gamma_{k}\right)$ which means that $i$ is not left dummy at $\gamma_{k}$. Let $\gamma_{k}<\xi<\beta_{k}$ be arbitrary. By definition of left coalition system $\bar{S} \in \mathcal{L}_{k}(\xi)$ and by condition $(3.13) \bar{S} \backslash\{i\} \subseteq\left\{j \in N \mid \tau_{k}\left(P_{j}\right) \leq \xi\right\} \notin \mathcal{L}_{k}(\xi)$. Therefore, there exists $T \subseteq \bar{S}$ such that $i \in T$ and $T \in \mathcal{L}_{k}^{m}(\xi)$. Hence $i$ is not a left-dummy agent at $\xi$, which shows (1).

\section{Conclusions}

We have characterized the maximal domains of preferences under which generalized median voter schemes are strategy-proof. The extent of these domains depends on the distribution of power among agents which is implied by each generalized median voter scheme. It is still an open question whether some form of single-peakedness is necessary for a domain of preferences to admit some strategyproof social choice function (not necessarily a generalized median voter scheme). An interesting partial answer is provided in Berga and Serizawa [5].

\section{References}

[1] Barberà, S., F. Gul, and E. Stacchetti (1993): "Generalized Median Voter Schemes and Committees". Journal of Economic Theory 61, 262-289.

[2] Barberà, S., J. Massó, and A. Neme (1997): "Voting under Constraints". Forthcoming in Journal of Economic Theory.

[3] Barberà, S., J. Massó, and S. Serizawa (1996): "Strategy-proof Voting on Compact Ranges". Universitat Autònoma de Barcelona, Working Paper 358.96.

[4] Barberà, S., H. Sonnenschein, and L. Zhou (1991): "Voting by Committees". Econometrica 59, 595-609. 
[5] Berga, D. and S. Serizawa (1996): "Maximal Domains for Strategy-proof Rules with One Public Good". Universitat Autònoma de Barcelona, Working Paper 353.96 .

[6] Border, K. and J. Jordan (1983): "Straightforward Elections, Unanimity and Phantom Voters", Review of Economic Studies 50, 153-170.

[7] Moulin, H. (1980): "On Strategy-proofness and Single Peakedness". Public Choice 35, 437-455.

[8] Peters, H., H. van der Stel, and H. Storken (1991): "On Uncompromisingness and Strategy-Proofness". Report M 91-15. ISSN.

[9] Serizawa, S. (1995): "Power of Voters and Domain of Preferences Where Voting by Committees Is Strategy-Proof". Journal of Economic Theory 67, 599608. 